\title{
Comparing SPEED and OSDI Questionnaires in a Non-Clinical Sample
}

\author{
Nauman Hashmani (iD) \\ Uzair Munaf ${ }^{2}$ \\ Aqsa Saleem (iD ${ }^{2}$ \\ Syed Owais Javed $\mathbb{D}^{2}$ \\ Sharif Hashmani ${ }^{1}$ \\ 'Ophthalmology and Visual Sciences, \\ Hashmanis Hospital, Karachi, Sindh, \\ Pakistan; ${ }^{2}$ Civil Hospital, Dow Medical \\ College, Karachi, Sindh, Pakistan
}

Purpose: To compare the performance of OSDI and SPEED questionnaires in a non-clinical sample in Pakistan.

Methods: SPEED and OSDI questionnaires were simultaneously administered to a sample of 600 participants in Pakistan with an average age of $31.0 \pm 10.1$ years.

Results: Mean SPEED scores were calculated according to the OSDI categories and were found to be $3.33 \pm 3.44,5.45 \pm 3.77,7.86 \pm 4.33$, and $9.39 \pm 4.56$ for the normal, mild, moderate, and severe groups, respectively. Using Cronbach's alpha, the total OSDI and SPEED scores were calculated to be 0.924 and 0.879 , respectively. A receiver operating curve was plotted, and the area under the curve (AUC) was estimated to be 0.791 . Using this curve, the cutoff score for the SPEED questionnaire was found to be $4.00(\mathrm{P}<0.001)$.

Conclusion: The SPEED questionnaire can be used as an effective alternative to the OSDI. Keywords: dry eye disease, dry eye syndrome, Cornea, aqueous deficiency, refractive surgery

\section{Introduction}

Many patient reported outcomes (PRO) questionnaires are currently available, including ocular surface disease index (OSDI; Allergan, Irvine, CA, USA) and standard patient evaluation of eye dryness (SPEED; Tear-Science, Morrisville, NC, USA). ${ }^{1}$ OSDI is a validated questionnaire that is frequently used in clinical trials ${ }^{2}$ as it provides a quick assessment of dry eye disease (DED) and its impact on patient's quality of life (QoL). ${ }^{3}$ SPEED, a recently introduced questionnaire for DED assessment is also now being widely used by eye care practitioners across the globe., ${ }^{4,5}$

A study conducted among university students in Ghana compared OSDI and SPEED in a non-clinical population, and established that both were equally good in distinguishing between symptomatic and asymptomatic patients. ${ }^{6}$ No such study has taken in Pakistan. The prevalence of DED in Pakistan is calculated to be as high as $43.6 \%$ to $64.4 \%{ }^{7}$ and therefore, having access to a greater number of tools can help in diagnosing this varied and large population. Thus, with this study we aim to compare the performance of OSDI and SPEED questionnaires and establish the reliability of SPEED by collecting data from a large, non-clinical population in Pakistan.

\section{Materials and Methods}

This cross-sectional study was conducted in Karachi after taking approval from Ethics Committee of the Hashmanis Hospital in agreement with the Declaration of Helsinki. Informed written consent was taken from all the study participants.
Correspondence: Nauman Hashmani Tel +92 2132781409

Email naumanhashmani@hashmanis.edu.pk 
Both questionnaires were administered simultaneously via a convenience sampling technique within a six-month period. Only those participants who were above 18 years of age, fluent in the English language and could understand the questionnaire being administered were included. We excluded participants with a prior history of intraocular surgery, meibomian gland dysfunction, ocular surface disease, an active infection, on topical medications, pregnant or breastfeeding. The study was explained clearly to every participant prior to administration. Any incomplete forms were discarded. The team of interviewers consisted of medical students who were given formal training.

OSDI consisted of 12 questions to assess the symptoms experienced by the patients in the last week. It was divided into 3 sections: the frequency of the experienced symptoms, their impact on vision-related QoL, and the presence of any environmental triggers. ${ }^{8}$ For every question, the participants had to choose amongst 5 options. Each was graded on a scale ranging from 0 to 4 : None of the time (0), some of the time (1), half of the time (2), most of the time (3), and all of the time (4). The final score was calculated on a scale of 0 to 100 , with higher scores representing greater disability. ${ }^{4}$ A score between 0 and 12 is considered normal, while a score between 13 and 22 represents a mild disease, between 23 and 32 is moderate DED, and any number between 33 and 100 is indicative of severe DED.

SPEED has four sections. ${ }^{4}$ The first three assess the presence, frequency, and severity of the following symptoms: (i) Dryness, Grittiness or Scratchiness, (ii) Soreness or Irritation, (iii) Burning or Watering, (iv) Eye fatigue, and the fourth reports the use of eye drops for lubrication. Additionally, the questionnaire helps record the changes in symptoms occurring as follows: (i) At this visit, (ii) Within past 72 hours, (iii) Within past 3 months. Furthermore, the question reporting the frequency of symptoms is graded on a 4-point scale: Never (0), Sometimes (1), Often (2), or Constant (3), whereas the question investigating the severity of the experienced symptoms is divided into a 5-point scale: No problems (0), Tolerable (1), Uncomfortable (2),
Bothersome (3), or Intolerable (4). Thus, by adding scores of the severity and frequency questions of SPEED questionnaire, we obtain a final SPEED score in the range of 0 to 28 .

\section{Statistical Analysis}

All statistical analyses were performed using the SPSS V.23.0 (SPSS, Chicago, IL) statistical package. A Pearson Correlation Coefficient was used. Cronbach's alpha was used as a measure of internal consistency to evaluate the reliability of OSDI and SPEED questionnaires, as well as their subsections. The receiver operating characteristic (ROC) curve was plotted to obtain a cutoff score for the SPEED questionnaire using OSDI, to help distinguish between symptomatic and asymptomatic subjects of the sample. This cutoff score was then further confirmed by finding an agreement between the OSDI and SPEED questionnaires using Cohen's Kappa.

\section{Results}

Out of the 611 questionnaires returned, 11 were incompletely filled, hence excluded from the analysis. The questionnaires of 600 participants were thus analyzed. The average age of the participants was $31.0 \pm 10.1$ years. The mean OSDI and SPEED scores of the participants were $22.4 \pm 19.6$ and $6.02 \pm 4.6$, respectively. We analyzed data from 200 males and 400 females.

The scores obtained by the OSDI questionnaire were used to classify all the participants into four categories. The mean SPEED scores for participants in each category were then calculated and recorded in Table 1.

The reliability of OSDI and SPEED questionnaires were measured using the Cronbach's alpha, as seen in Tables 2 and 3, respectively. The final score of the OSDI questionnaire scored relatively better, however the individual subsections of both questionnaires had similar performance.

A scatter plot was constructed to observe the correlation between the two questionnaires. Coefficient of Correlation was calculated to be $\mathrm{R}=0.5110 ; \mathrm{P}<0.001$,

Table I Mean Speed Score by OSDI Categorization

\begin{tabular}{|l|l|l|l|}
\hline OSDI Scores & OSDI Category & Participants (N) & SPEED Score (Means \pm SD) \\
\hline $0-12$ & Normal & 224 & $3.33 \pm 3.44$ \\
$12-22$ & Mild & 134 & $5.45 \pm 3.77$ \\
$23-32$ & Moderate & 88 & $7.86 \pm 4.33$ \\
$33-100$ & Severe & 154 & $9.39 \pm 4.56$ \\
\hline
\end{tabular}

Abbreviations: OSDI, ocular surface disease index; N, number; SPEED, standard patient evaluation of eye dryness; SD, standard deviation. 
Table 2 Cronbach's Alpha of OSDI Questionnaire and Its SubSections

\begin{tabular}{|l|c|}
\hline Category & Cronbach's Alpha \\
\hline OSDI & 0.924 \\
Ocular & 0.790 \\
Vision Related & $0.88 \mathrm{I}$ \\
Environmental & 0.864 \\
\hline
\end{tabular}

Abbreviation: OSDI, ocular surface disease index.

Table 3 Cronbach's Alpha of SPEED Questionnaire and Its SubSections

\begin{tabular}{|l|c|}
\hline Category & Cronbach's Alpha \\
\hline SPEED & 0.879 \\
Frequency & 0.780 \\
Severity & 0.833 \\
\hline
\end{tabular}

Abbreviation: SPEED, standard patient evaluation of eye dryness.

showing a moderate correlation between the total scores of both the questionnaires. This is shown in Figure 1.

A receiver operating characteristic (ROC) curve for the SPEED questionnaire was also plotted, and the area under the curve (AUC) was estimated to be 0.791, as shown in Figure 2. The cut-off score for SPEED questionnaire with the maximum sum of sensitivity and specificity was found to be 4.00 . This can be seen in Table 4 .

The inter-rater reliability of the SPEED questionnaire at a cut-off value of 4.00 was found using Cohen's Kappa highlighting moderate agreement between both SPEED and OSDI questionnaires for the diagnosis of DED. This can be seen in Table 5 .

\section{Discussion}

We performed a cross-sectional analysis using the OSDI and SPEED questionnaires to understand if, like OSDI, SPEED can also be used to assess dry eye symptoms. Other studies have used various methods to determine the validity of SPEED, including a psychometric

Table 4 Cutoff Values for SPEED

\begin{tabular}{|l|c|c|}
\hline Cutoff & Sensitivity (\%) & Specificity (\%) \\
\hline 3.00 & 80.59 & 61.16 \\
4.00 & 73.40 & 70.54 \\
5.00 & 67.02 & 77.23 \\
6.00 & 58.24 & 84.82 \\
\hline
\end{tabular}

Abbreviation: SPEED, standard patient evaluation of eye dryness.

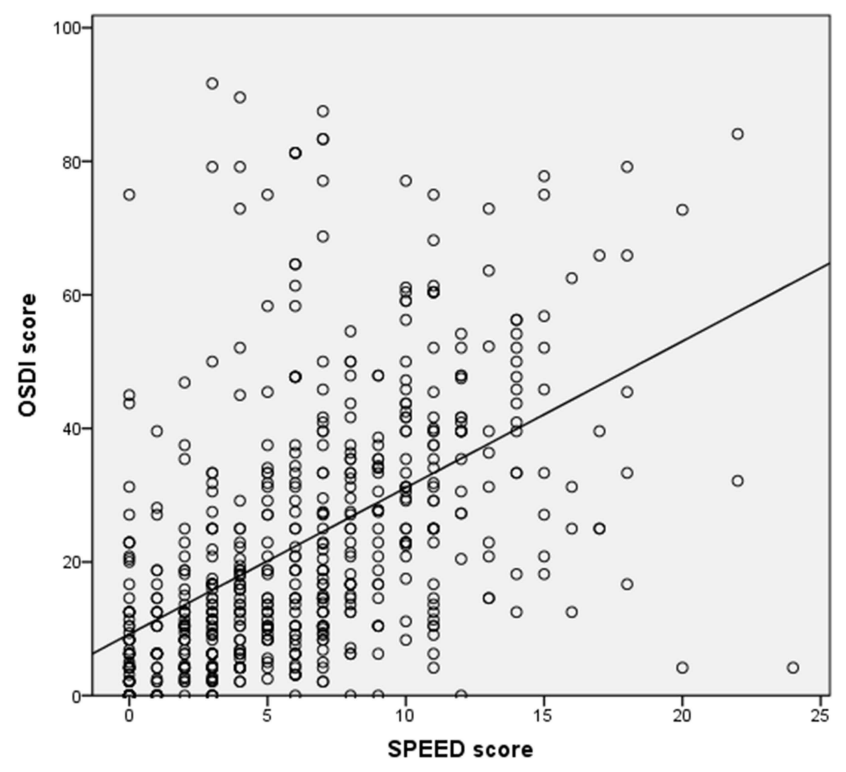

Figure I Scatter plot of OSDI against SPEED scores. Abbreviations: SPEED, standard patient evaluation of eye dryness; OSDI, ocular surface disease index.

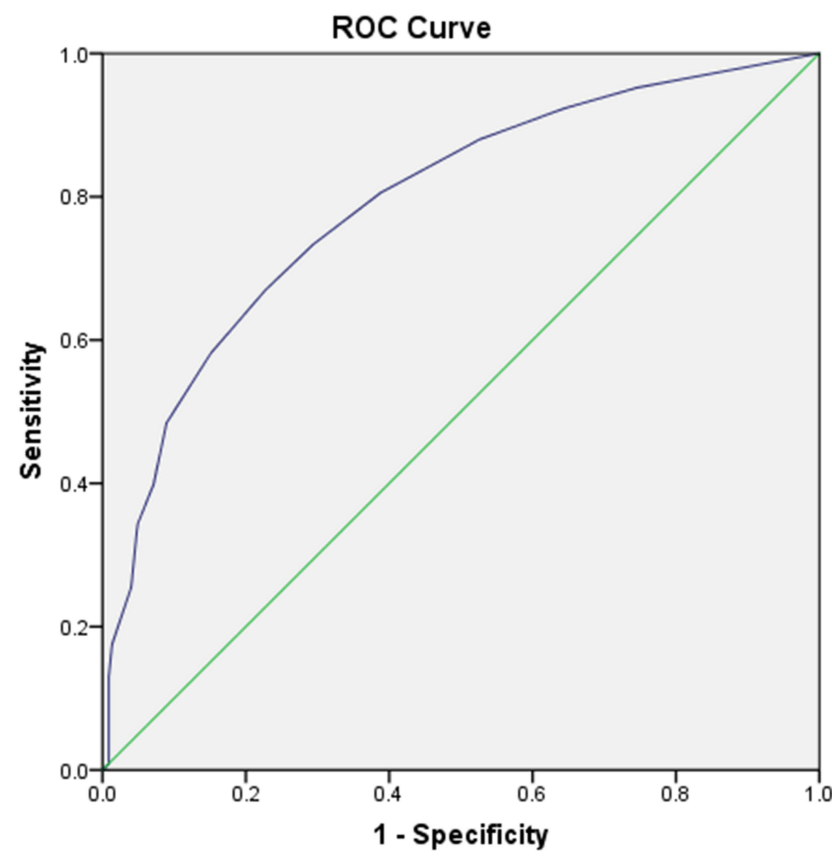

Diagonal segments are produced by ties.

Figure 2 Area under the curve for SPEED.

Abbreviation: OSDI, ocular surface disease index.

analysis. $^{4,6,9}$ Additionally, the questionnaire has been translated to other languages. ${ }^{10}$

DED is a multifactorial disease with poor correlation between signs, symptoms, and clinical tests. ${ }^{11-13}$ Despite this, patient reported outcomes (PRO) questionnaires are widely in use. These PRO questionnaires have helped 
Table 5 Cohen's Kappa with Various SPEED Cutoff Values

\begin{tabular}{|l|c|l|c|c|}
\hline Cutoff & Cohen's Kappa & P-value & Sensitivity (\%) & Specificity (\%) \\
\hline 3.00 & 0.423 & $<0.001$ & 80.59 & 61.16 \\
4.00 & 0.426 & $<0.001$ & 73.40 & 70.54 \\
5.00 & 0.415 & $<0.001$ & 67.02 & 77.23 \\
6.00 & 0.388 & $<0.001$ & 58.24 & 84.82 \\
\hline
\end{tabular}

Abbreviation: SPEED, standard patient evaluation of eye dryness.

remarkably in the diagnosis of DED, as well as in grading the severity and frequency of its symptoms. ${ }^{14}$ These symptoms heavily impact the patients' QoL, ${ }^{15,16}$ and recognizing these symptoms aids in providing holistic care to the patient.

OSDI is a clinically validated and widely used PRO questionnaire that has a multitude of advantages. It has been used to monitor treatment response for DED, ${ }^{17}$ and to rule in possible clinical signs. ${ }^{18}$ Moreover, it has also been used to validate newly introduced diagnostic tests that could potentially help in the assessment of the disease. ${ }^{19}$ Similarly, in this study, OSDI has been used to test the reliability of SPEED questionnaire; SPEED performs comparably in terms of the internal consistency. OSDI showed a slightly better internal consistency which is explained by the fact that any questionnaire with a greater number of items will have an exaggerated Cronbach's Alpha, regardless of its true internal consistency. ${ }^{20}$

Furthermore, a mean SPEED value was calculated for every OSDI category. It was observed that the SPEED scores increased with the increasing degree of disease severity. This association could suggest that SPEED could also be used to assess disease severity, like the OSDI. Furthermore, the inter-rater reliability was calculated using Cohen's Kappa which showed moderate agreement.

This study shows that the SPEED questionnaire is not only comparable to OSDI in several aspects but is also reliable in distinguishing between symptomatic and asymptomatic participants. Therefore, the SPEED questionnaire can also be used as an effective tool for dry eye symptom assessment in current ophthalmic practice. However, this study does not distinguish participants based on their usage of contact lens, or any other predisposing eye diseases, and hence we cannot conclude for certain how SPEED will perform in this demographic. Furthermore, only those individuals that could speak English were included and therefore a large subset of people was missed.

\section{Acknowledgments}

No acknowledgments to make.

\section{Funding}

This research was funded by the Hashmanis Foundation.

\section{Disclosure}

The authors report no conflicts of interest in this work.

\section{References}

1. Shiraishi A, Sakane Y. Assessment of dry eye symptoms: current trends and issues of dry eye questionnaires in Japan. Investig Ophthalmol Vis Sci. 2018;59(14):DES23-DES28. doi:10.1167/ iovs. 18-24570

2. Yazıc1 A, Sarı E, Ayhan E, et al. The effect of low-dose aspirin on dry eye parameters and ocular surface disease index questionnaire. $J$ Ocul Pharmacol Ther. 2018;34(3):256-259. doi:10.1089/jop.2017.0064

3. Schiffman RM, Christianson MD, Jacobsen G, Hirsch JD, Reis BL. Reliability and validity of the ocular surface disease index. Arch Ophthalmol. 2000;118(5):615-621. doi:10.1001/archopht.118.5.615

4. Pucker AD, Dougherty BE, Jones-Jordan LA, Kwan JT, Kunnen CME, Srinivasan S. Psychometric analysis of the SPEED questionnaire and CLDEQ-8. Investig Ophthalmol Vis Sci. 2018;59 (8):3307-3313. doi:10.1167/iovs.18-24016

5. Dell SJ, Gaster RN, Barbarino SC, Cunningham DN. Prospective evaluation of intense pulsed light and meibomian gland expression efficacy on relieving signs and symptoms of dry eye disease due to meibomian gland dysfunction. Clin Ophthalmol. 2017;11:817-827. doi:10.2147/OPTH.S130706

6. Asiedu K, Kyei S, Mensah SN, Ocansey S, Abu LS, Kyere EA. Ocular surface disease index (OSDI) versus the standard patient evaluation of eye dryness (SPEED): a study of a nonclinical sample. Cornea. 2016;35(2):175-180. doi:10.1097/ ICO.0000000000000712

7. Hashmani N, Mustafa FG, Tariq MA, et al. Distribution and correlation of ocular surface disease index scores in a non-clinical population: the Karachi ocular surface disease study. Cureus. 2020;12(7): e9193-e9193.

8. Dougherty BE, Nichols JJ, Nichols KK. Rasch analysis of the ocular surface disease index (OSDI). Investig Ophthalmol Vis Sci. 2011;52 (12):8630-8635. doi:10.1167/iovs.11-8027

9. Ngo W, Situ P, Keir N, Korb D, Blackie C, Simpson T. Psychometric properties and validation of the standard patient evaluation of eye dryness questionnaire. Cornea. 2013;32(9):1204-1210. doi:10.1097/ ICO.0b013e318294b0c0

10. Facchin A, Boccardo L. Italian translation, validation, and repeatability of Standard Patient Evaluation of Eye Dryness (SPEED) Questionnaire. Contact Lens Anterior Eye. 2021;11:101497. doi:10.1016/j.clae.2021.101497 
11. Vehof J, Sillevis Smitt-Kamminga N, Nibourg SA, Hammond CJ. Predictors of discordance between symptoms and signs in dry eye disease. Ophthalmology. 2017;124(3):280-286. doi:10.1016/j. ophtha.2016.11.008

12. Ong ES, Felix ER, Levitt RC, Feuer WJ, Sarantopoulos CD, Galor A. Epidemiology of discordance between symptoms and signs of dry eye. Br J Ophthalmol. 2018;102(5):674-679. doi:10.1136/bjophthalmol-2017-310633

13. Sullivan BD, Crews LA, Messmer EM, et al. Correlations between commonly used objective signs and symptoms for the diagnosis of dry eye disease: clinical implications. Acta Ophthalmol. 2014;92 (2):161-166. doi:10.1111/aos.12012

14. Zeev MSB, Miller DD, Latkany R. Diagnosis of dry eye disease and emerging technologies. Clin Ophthalmol. 2014;8:581-590.

15. Benítez-del-Castillo J, Labetoulle M, Baudouin C, et al. Visual acuity and quality of life in dry eye disease: proceedings of the OCEAN group meeting. Ocular Surface. 2017;15:169-178. doi:10.1016/j. jtos.2016.11.003
16. Uchino M, Schaumberg DA. Dry eye disease: impact on quality of life and vision. Curr Ophthalmol Rep. 2013;1(2):51-57. doi:10.1007/ s40135-013-0009-1

17. Oh SH, Lyu B, Yim HB, Lee NY. Lower lid laxity is negatively correlated with improvement of the ocular surface disease index in dry eye treatment. Curr Eye Res. 2016;41(2):165-170. doi:10.3109/ 02713683.2015.1015142

18. Pult H, Bandlitz S. Lid-parallel conjunctival folds and their ability to predict dry eye. Eye Contact Lens. 2018;44:S113-S119. doi:10.1097/ ICL.0000000000000435

19. Zhu K, Xie W, Ying J, Yao Y. Evaluation of tear film and meibomian gland function in dry eye patients using Keratograph 5M. Zhejiang Da Xue Xue Bao Yi Xue Ban. 2016;45(4):422-428.

20. Bland JM, Altman DG. Statistics notes: Cronbach's alpha. BMJ. 1997;314(7080):572. doi:10.1136/bmj.314.7080.572
Clinical Ophthalmology

\section{Publish your work in this journal}

Clinical Ophthalmology is an international, peer-reviewed journal covering all subspecialties within ophthalmology. Key topics include: Optometry; Visual science; Pharmacology and drug therapy in eye diseases; Basic Sciences; Primary and Secondary eye care; Patient Safety and Quality of Care Improvements. This journal is indexed on PubMed

Submit your manuscript here: https://www.dovepress.com/clinical-ophthalmology-journal
Dovepress

Central and CAS, and is the official journal of The Society of Clinical Ophthalmology (SCO). The manuscript management system is completely online and includes a very quick and fair peer-review system, which is all easy to use. Visit http://www.dovepress.com/ testimonials.php to read real quotes from published authors. 